\title{
HIGH-DIMENSIONAL IMAGE INDEXING BASED ON ADAPTIVE PARTITIONING AND VECTOR APPROXIMATION
}

\author{
Guang-Ho Cha \\ Department of Multimedia Engineering, Tongmyong University of Information Technology, \\ Busan 608-711, South Korea. \\ ghcha@tit.ac.kr
}

Abstract: In this paper, we propose the $L P C^{+}$-file for efficient indexing of high-
dimensional image data. With the proliferation of multimedia data, there is an
increasing need to support the indexing and retrieval of high-dimensional im-
age data. Recently, we developed the LPC-file (Cha et al. 2002) for indexing
high-dimensional data based on vector approximation. The LPC-file gives
good performance especially when the dataset is uniformly distributed. How-
ever, compared with for the uniformly distributed dataset, its performance de-
grades when the dataset is clustered. Real image datasets are often strongly
clustered and the dimensions of the feature vectors are usually correlated. We
improve the performance of the LPC-file for the strongly clustered image data-
set. The basic idea is to adaptively partition the data space to find subspaces
with high-density clusters and to assign more bits to them to increase the dis-
criminatory power of the vector approximation. An empirical evaluation shows
that the LPC ${ }^{+}$-file results in significant performance improvements for real
image datasets strongly clustered.

Key words: $\quad \mathrm{LPC}^{+}$-file, high-dimensional indexing, similarity search, nearest neighbor search, multimedia database

\section{INTRODUCTION}

Image databases often represent the image objects as vectors of $d$ numeridc features, and access them via the feature vectors and similarity measures. Feature vectors can be viewed as points in a $d$-dimensional vector space and the similarity measure can be viewed as a measure of distance 
within that space. An important problem in such a system is to find the $k$ most similar images to a given sample image. This problem is typically solved by first mapping the sample image to its corresponding feature vector and then finding the $k$ nearest neighbors (NNs) of the vector. The basic issue then is how to efficiently find the $k$ NNs.

For applications where the vectors have low or medium dimensionalities (e.g., less than 10), existing multidimensional indexing methods (MIMs), such as the $\mathrm{R}^{*}$-tree (Beckmann et al. 1990), the X-tree (Berchtold et al. 1996), the HG-tree (Cha \& Chung 1998), and the SR-tree (Katayama \& Satoh 1997), can be usefully employed to solve the problem. So far, however, there is no effective solution to this problem for the applications in which the vectors have high dimensionalities, say over 100 . In fact, for a high dimensionality, in theory or in practice, the performance of existing MIMs degenerates to being worse than that of the brute-force sequential scan that compares the query object to each data object (Arya et al. 1998) (Indyk \& Motwani 1998) (Kushilevitz et al. 1998) (Weber et al. 1998). So the main issue is to overcome the curse of dimensionality (Niblack et al. 1993) - a phenomenon that the performance of indexing methods degrades drastically as the dimensionality increases. With this urgent need, recently, we developed a new vector approximation-based indexing method called the LPC (Local Polar Coordinate)-file for the $k$-nearest neighbor $(k-\mathrm{NN})$ search. The LPCfile significantly improves the search performance for large collections of high-dimensional vectors compared with the sequential scan and the VA-file (Weber et al. 1998). Although the LPC-file provides significant improvements compared with current techniques, it suffers the performance degradation when the dataset is highly clustered because it employs the simple space partitioning and the uniform bit allocation strategy for the partitioned cells. These strategies are appropriate for only uniform datasets. However, real image datasets are not uniformly distributed but highly clustered and skewed. In this paper, we discuss the problem to efficiently index high-dimensional image datasets.

\section{RELATED WORK}

Various approaches have been tried for the $k$-NN search in database systems. Intuitively, it is natural to use MIMs to speed up the $k$-NN search because objects are often represented by points in a multidimensional data space. However, most MIMs are defeated by the high dimensionality. MIMs work by partitioning the data space, clustering data according to partitions, and using the partitions to prune the search space for queries. Unfortunately, while MIMs generally perform well at low dimensional data spaces, their 
performance degrades drastically as the dimensionality increases. It is very unlikely that existing MIMs can prune some search space in high-dimesional data spaces. Because of the sparsity of the data space caused by the high dimensionality, the distance $k-N N^{d i s t}(q)$ between the query point $q$ and its $k$ th NN may become far larger than the length of each dimension of the data space. As a result, the sphere $k-N N^{\text {sphere }}(q)$ with center $q$ and radius $k$ $N N^{d i s t}(q)$ may intersect every partition in the data space, and thus the $k$-NN search may visit every partition (node) in an index. Motivated by the disadvantages of the state-of-the-art MIMs for high-dimensional data spaces, more recently, other kinds of approaches have been investigated to overcome the dimensionality curse. They are classified into four categories:

- Dimensionality reduction approach,

- Approximate nearest neighbor approach,

- Multiple space-filling curves approach,

- Vector approximation-based approach.

The dimensionality reduction approaches (Chakrabarti \& Mehrotra 2000) (Kanth et al. 1998) (Lin et al. 1994) first condense most of information in a dataset to a few dimensions by applying the Singular Value Decomposition, the Discrete Cosine Transform or the Discrete Wavelet Transform. The data in the few condensed dimensions are then indexed to support fast retrieval. While the methods based on the DR approach provide a solution to the dimensionality curse, they have several drawbacks: (1) Dimensionality reduction is inevitably accompanied by the loss of precision of query results; (2) They are not readily applicable to dynamic databases because the transform has to be computed a priori on the entire data set and the computation is expensive; (3) The dimensionality may be still so high even after it is reduced that the MIMs cannot be used for the dataset.

The idea behind the approximate nearest neighbor (ANN) approaches (Arya et al. 1998) (Indym \& Motwani 1998) (Kushilevitz et al. 1998) is to retrieve $k$ ANNs faster within a given error bound $\varepsilon$ instead of retrieving exact $k$ NNs. Given a query point $q$ and a distance error $\varepsilon>0$, a point $p$ is a $(1+\varepsilon)$-approximate nearest neighbor of $q$ such that for any other database point $\boldsymbol{p}^{\prime}$

$$
\|\boldsymbol{q}-\boldsymbol{p}\| \leq(1+\varepsilon)\left\|\boldsymbol{q}-\boldsymbol{p}^{\prime}\right\|
$$

The multiple space-filling curves approach $[14,17]$ is also a kind of approximate approach in the sense that it gives up some accuracy of query results. This approach orders the $d$-dimensional space in many ways, with a set of space-filling curves such as Hilbert curves, each constituting a mapping from $R^{d} \rightarrow R^{1}$. This mapping gives a linear ordering of all points in the data set. Therefore, when a query point is mapped to the space-filling curve, one can perform a range search for nearby points along the curve to 
find near neighbors in the data space. However, due to the nature of the $R^{d}$ $\rightarrow R^{1}$ mapping, some near neighbors of the query point may be mapped far apart along a single curve. To make sure that these points are not overlooked, multiple space-filling curves are used, based on different mappings from $R^{d}$ $\rightarrow R^{1}$. The set of candidate nearest neighbors is formed from the union of sets of points in small neighborhoods (ranges) from all of the curves.

Although this approach improves the performance of the $k$-NN search, it is possible that some near-neighbors may be omitted during a search. To improve the chances of finding all $k$ NNs, more space-filling curves have to be used or a larger neighborhood on each curve has to be scanned. However, they diminish the benefits of the approximate approach since both of these methods increase the size of the candidate set.

The vector approximation approach overcomes the dimensionality curse by filtering the feature vectors so that only a small fraction of them must be visited during a search. Among the approaches that aim at overcoming the dimensionality curse, this approach is the only one that outputs the exact $k$ NNs. The VA-file (Weber et al. 1998) and the LPC-file (Cha et al. 2002) are classified into this category. The VA-file divides the data space into $2^{b}$ rectangular cells where $b$ denotes the total number of bits specified by the user. Each cell has a bit representation of length $b$ which approximates the data points that fall into a cell. The VA-file itself is simply an array of these bit vector approximations of data points.

The $k$-NN search in a VA-file consists of two phases. In the first phase, the set of all vector approximations is scanned sequentially and lower bound $d_{\min }$ and upper bound $d_{\max }$ on the distance of each cell to the query vector are computed. In this phase, if an approximation is encountered such that its $d_{\min }$ exceeds the $k$-th smallest upper bound found so far, the corresponding vector can be eliminated safely since $k$ better candidates already exist. At the end of this phase, the vectors with the smallest bounds are found to be the candidates for $k \mathrm{NNs}$ of the query. In the second phase, the algorithm refines the candidate set by visiting real vectors themselves in the increasing order of $d_{\min }$. This phase ends when an approximation is encountered whose $d_{\min }$ exceeds or equals the $k$-th actual NN distance in the answer set, and the final $k$ nearest vectors in the answer set are the search result.

The LPC-file aims at improving the filtering power of the vector approximation with a minimum number of additional bits. The LPC-file partitions the vector space into hyper-square cells and approximates vectors using the polar coordinates on the partitioned cells. The approximation $a$ for a vector $\boldsymbol{p}$ in the LPC-file is generated as follows. The first step is to assign the same number of bits $b$ to each dimension and to divide the whole data space into $2^{b d}$ cells, where $d$ is the number of dimensions. Thus the length of each side of a cell is the same. Typically $b$ is a small integer between 4 and 8 


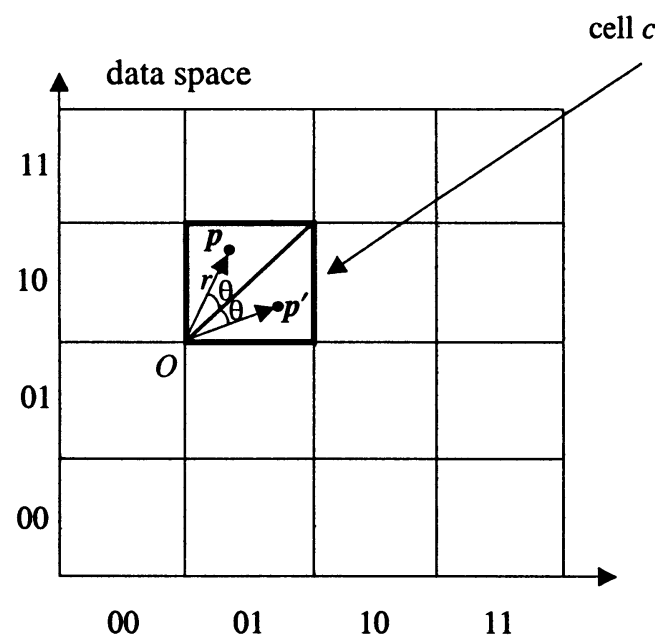

(a) 2-dimensional space cell $c$ for vector $\boldsymbol{p}$

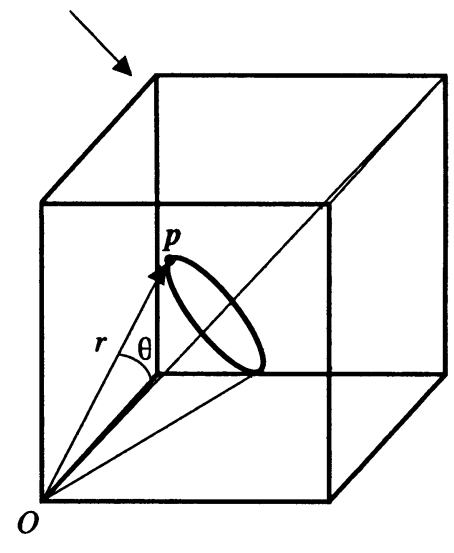

(b) 3-dimensional space

Figure 1. Vector $\boldsymbol{p}$ and its approximation in the LPC-file

depending on the dimensionality and the data distribution. The cell is simply represented by the concatenation of the binary bit patterns for each dimension in turn. Figure 1(a) shows an example in a 2-dimensional space: the cell $c$ is represented by the sequence of bits $(0110)$ where $d=2$ and $b=2$.

The second step is to represent the vector $p$ using the polar coordinates $(r$, $\theta$ ) within the cell in which $p$ lies. As illustrated in Figure 1(a), the local origin $O$ of each cell is determined as the lower left corner of the cell. The distance $r$ of the polar coordinate is computed by the distance between the local

origin $O$ and the vector $p$. The angle $\theta$ is computed by the angle between the vector $\boldsymbol{p}$ and the diagonal from the local origin to the opposite corner. As a result of this approximation, the vector $\boldsymbol{p}$ is represented by the triplet $\boldsymbol{a}=<c$, $r, \theta>$, where $c, r, \theta$ denote the approximation cell, the distance and the angle of $\boldsymbol{p}$ based on the local origin $O$, respectively. The compactness of the LPCfile vector approximation improves the search performance.

\section{LPC ${ }^{+}$-FILE FOR IMAGE DATA}

The current vector approximation approach has an implicit assumption that it is very unlikely that several points lie in the same cell since the number of cells $2^{b d}$ is far larger than the size of a database, where $b$ is the number of bits assigned to each dimension and $d$ is the dimensionality. Actually, the vector approximation approach benefits from the sparseness of the data space as opposed to the partitioning or clustering methods (i.e., the tradi- 
tional MIMs). However, if the data points are highly clustered, the probability that a certain cell includes several points increases, and therefore those vectors may use the same approximation. This means that the discriminatory power of the approximation decreases and thus candidates are less eliminated, and ultimately more disk accesses occur during the search.

Figures 2 and 3 show the vector selectivity comparison between random and real image datasets during the filtering and the refinement phases of the vector approximation approach, respectively. The vector selectivity is a good performance estimator because the performance of the $k$-NN search depends largely on the number of disk blocks visited and it is affected by the vector selectivity. The first phase vector selectivity means the ratio of candidates not eliminated after the first filtering phase to the total number of vectors. The second phase vector selectivity is the ratio of real vectors visited to the total number of vectors. In Figure 2, the $x$-axis represents the datasets and query types used in the experiment and the $y$-axis represents the percentage of remaining vectors after the filtering phase (i.e., the first phase vector selectivity). The cluster query means that the query vector is selected from the real image dataset itself, and the random query means that the query vector is selected randomly. In the experiment, we used 13,724 256-dimensional images of U.S. stamps and photos: Stamps often come in series (e.g., states, birds, flowers) with common colors and related designs, and the U.S. Post Office has often used similar colors for many long-running stamps. As a result, this real image dataset shows strongly clustered distribution. The random dataset used in the experiment also consists of 256-dimensional 13,724 random vectors. For the random dataset, 1,000 random $10-\mathrm{NN}$ queries were posed, and for the real image dataset, 1,000 random 10-NN queries and 1,000 cluster $10-\mathrm{NN}$ queries were posed, and their results were averaged.

In the case of the LPC-file in Fig. 2(a), the experimental result shows that, after the first phase filtering, the number of remaining vectors for a real image dataset is 2.66 to 4.68 times more than that for a random dataset. Similarly, in the case of the VA-file in Fig. 2(b), after the first phase filtering, the number of remaining vectors for a real image dataset is 2.34 to 16.57 times more than that for a random dataset. These mean that, compared with the random dataset, for the clustered dataset, the filtering power of the current vector approximation approach decreases drastically.

For the second refinement phase, in the case of the LPC-file as shown in Fig. 3, the number of real vectors visited for a real image dataset is 2.43 to 6.49 times more than that for a random dataset. In the VA-file, the number of real vectors visited for a real image dataset is 2.02 to 76.67 times more than that for a random dataset. These mean that the $\mathrm{I} / \mathrm{O}$ cost occurred during the $k$-NN search for the clustered dataset is much higher than that for the random dataset. 


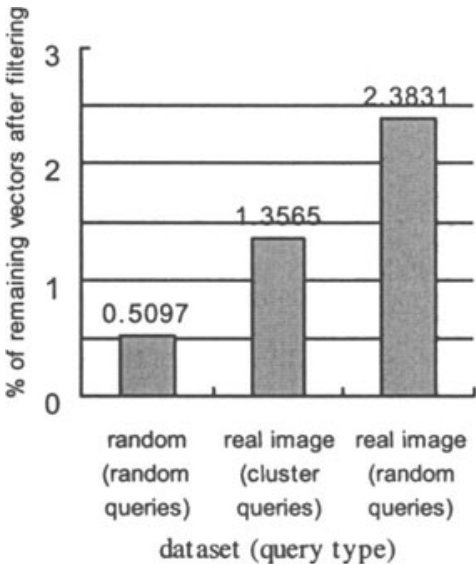

(a) LPC-file

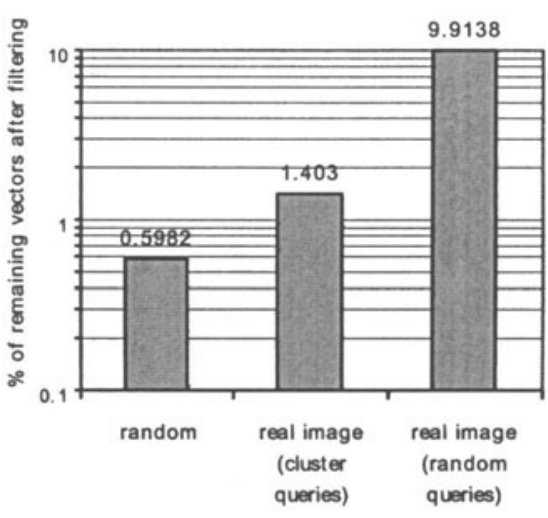

dataset (query type)

Figure 2. Selectivity comparison between random and real image datasets in the first phase (filtering)

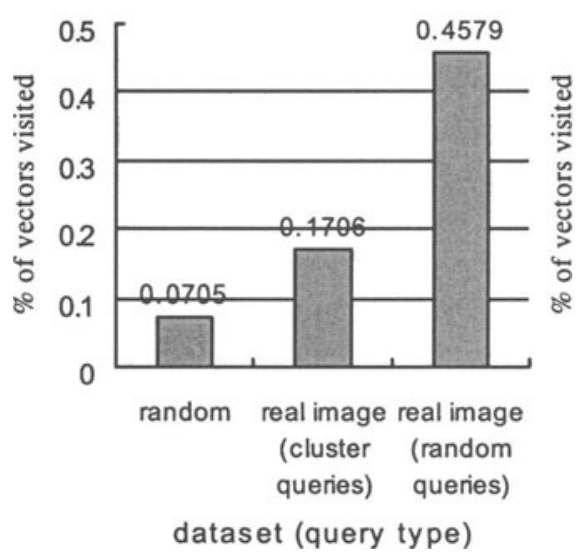

(a) LPC-file

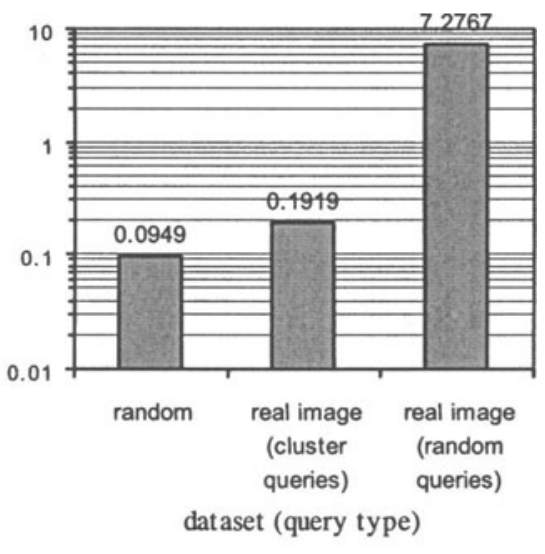

(b) VA-file

Figure 3. Selectivity comparison between random and real image datasets in the second phase (refinement)

This performance degeneration for the clustered dataset is a common problem of the current vector approximation approach. The reason for the performance degeneration is because bits are uniformly allocated to each partitioned cell while the data distribution is not uniform and the density of each cell varies greatly. The way to overcome this problem is to adaptively assign bits to each cell according to the data statistics of the cell. This is the focus of the $\mathrm{LPC}^{+}$-file. 


\subsection{Adaptive Partitioning of Data Space}

The $\mathrm{LPC}^{+}$-file uses a density-based approach to determine the number of bits to allocate to a cell. To approximate the density of the data points, the $\mathrm{LPC}^{+}$-file partitions the data space into hyper-square cells and finds the number of points that lie inside each cell of the partition. To accomplish this, like in the LPC-file, initially we assign $b$ bits equally to each dimension $j, j$ $\in\{1, \ldots, d\}$, and divide the whole data space into $2^{b d}$ cells, where $d$ is the number of dimensions and $b$ is typically a small integer between 4 and 8 depending on the dimensionality and the data distribution. Since each cell has the same volume, the number of points inside it can be used to approximate the density of the cell. The vast majority of the partitions are empty since the number of partitions $2^{\text {bd }}$ is much larger than the number of points in a dataset. In addition, for a real image dataset, most points lie inside some partitions due to the high clustering property of the image dataset.

The selectivity of a cell is defined to be the fraction of data points in the cell to the total data points. We call a cell $\mathrm{c}$ dense if the selectivity of $c$ is greater than $\tau$, where the density threshold $\tau$ is an input parameter. Otherwise, the cell is called sparse. We call a dense cell a cluster and call the points that lie inside the sparse cells outliers.

Once the sparse cells are found, the outliers lying inside the sparse cell are represented by approximation $\langle c, r, \theta\rangle$, where $c, r$ and $\theta$ are the same as the LPC-file's. The cell $c$ of the initial outliers is represented by the bit pattern of the partition itself. That is, the initial outliers are represented by $b d$ bits. Clusters are further partitioned into $2^{d}$ sub-cells until the points inside them become outliers. Outliers generated from a common partition share the bit representation of the partition. Thus, the cell of each outlier is represented by the common bit patterns of the partition to which the cell belongs and its own $d$ bits for the cell it belongs to. In other words, the outliers generated from a common partition are represented by only $d$ bits and the common bit pattern is shared among outliers. In effect, this greatly reduces the number of bits to represent the cell, and therefore the I/O cost for reading approximations can be reduced. Figure 4 shows the number of disk accesses for reading the approximation files for the $\mathrm{LPC}^{+}$-file, the LPC-file, and the VA-file from the same experiments described above. In other words, this illustrates the $\mathrm{I} O$ cost for the first phase of the $k$-NN search in the vector approximation approach. During the experiment, the size of a page used was $4 \mathrm{~KB}$. The number of disk accesses of the LPC ${ }^{+}$-file is almost a half of those of the LPC-file and the VA-file. This means that, for the $\mathrm{LPC}^{+}$-file, the number of bits used to represent the cell approximation is a half of those of the LPC-file and the VA-file. 


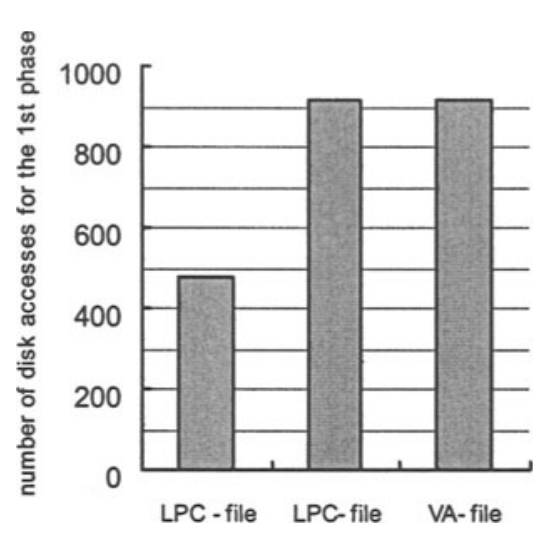

Figure 4. Comparison of the number of disk accesses during the first phase of the search data space

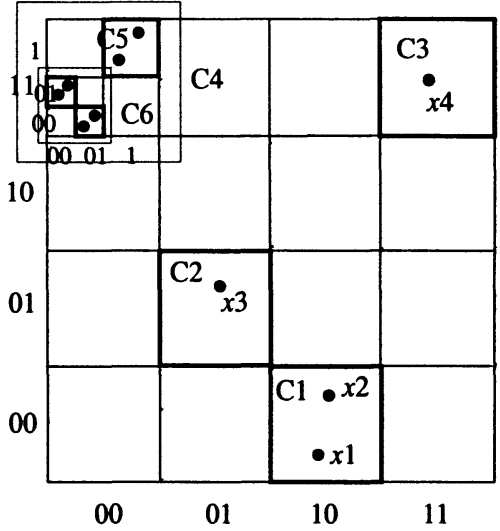

Figure 5. $\mathrm{LPC}^{+}$-file's non-uniform partition of the data space

Example 1: We assume that the dimensionality is 2, the number of bits initially assigned to each dimension is 2 , and the density threshold $\tau$ is $2 / N$, where $N$ is the total number of points in a database. In Figure 5, the twodimensional space has been initially partitioned by $4 \times 4$ cells by using 2 bits per dimension. Outliers, i.e., data points fallen into a cell $c_{i}$ whose selectivity is less than or equal to $\tau$, are approximated by $\left\langle c_{i}, r, \theta\right\rangle$. Points $x 1, x 2, x 3$, and $x 4$ are approximated by the cell $c_{i}$ in which they lie and their polar coordinates in $c_{i}$. Clusters, i.e., the cells whose selectivity is greater than $\tau$, e.g., cell $\mathrm{C} 4$, are further divided into $2^{2}$ sub-cells. The cell $\mathrm{C} 4$ is subdivided into 4 cells and the points in cell $\mathrm{C} 5$ are approximated by $\left\langle c_{5}, r, \theta\right\rangle$. The cell $\mathrm{C} 6$ is further divided into smaller cells and finally all points are approximated. The cell representations of the cells $\mathrm{C} 1, \mathrm{C} 2$, and C3 are (10 00), (01 01), and (11 $11)$, respectively. The points in the cell $\mathrm{C} 5$ have the cell representation of ( 1 $1)$ and a common cell representation (00 11). The two cells within the cell C6 have the cell representations ( 01$)$ and (1 0), respectively, with a common cell representation $(000110)$ for C6. Since the real image data tend to cluster in certain cells, in fact, there are many points that share a common cell representation. This reduces the cost of disk $\mathrm{V} / \mathrm{O}$ as shown in Figure 4.

\subsection{Algorithm}

The adaptive partitioning algorithm first starts partitioning the data space with $b$ bits per dimension. From this initial partitioning, outliers and clusters are determined, and the cells of the outliers are represented by $b d$ bits. The clusters are further partitioned to find appropriate number of bits to represent them and subsequent outliers are found and approximated. The following algorithm performs the partitioning of a cluster. The function MakeOutliers 
collects outliers on a given level and approximates them by using the LPCfile approximation.

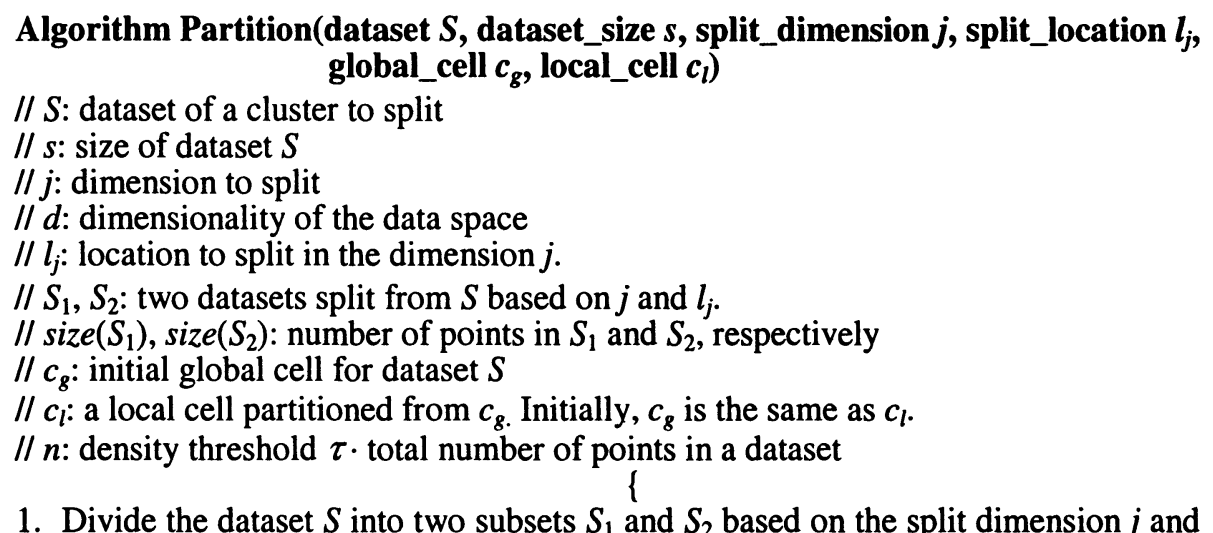
the split location $l_{j}$.

2. If $\left(\operatorname{size}\left(S_{1}\right)>0\right)\{$ If $(j<d)$ \{

2.1 Determine a new split location $l_{j+1}$ based on the local cell $c_{l}$ and new split dimension $j+1$.

2.2 Adjust the local cell $c_{l}$ based on the split.

\}

2.3 Invoke Partition $\left(S_{1}, \operatorname{size}\left(S_{1}\right), j+1, l_{j+1}, c_{g}, c_{l}\right)$.

Else \{

If $\left(\operatorname{size}\left(S_{1}\right)<n\right)$ Else \{

Invoke MakeOutliers $\left(S_{1}, \operatorname{size}\left(S_{1}\right), c_{g}\right)$.

2.1 Determine a new split location $l_{1}$ based on the local cell $c_{l}$ and the first dimension 1.

2.2 Invoke Partition $\left(S_{1}, \operatorname{size}\left(S_{1}\right), 1, l_{1}, c_{l}, c_{l}\right)$.

\} \} \}

3. If $\left(\operatorname{size}\left(S_{2}\right)>0\right)\{$

If $(j<d)\{$

3.1 Determine a new split location $l_{j+1}$ based on the local cell $c_{l}$ and the new split dimension $j+1$.

3.2 Adjust the local cell $c_{l}$ based on the split.

\}

3.2 Invoke Partition $\left(S_{2}, \operatorname{size}\left(S_{2}\right), j+1, l_{j+1}, c_{g}, c_{l}\right)$.

Else \{

If $\left(\operatorname{size}\left(S_{1}\right)<n\right)$ Else \{

Invoke MakeOutliers $\left(S_{2}, \operatorname{size}\left(S_{2}\right), c_{g}\right)$.

3.1 Determine a new split location $l_{1}$ based on the local cell $c_{l}$ and the first dimension 1.

3.2 Invoke Partition $\left(S_{2}, \operatorname{size}\left(S_{2}\right), 1, l_{1}, c_{l}, c_{l}\right)$. 


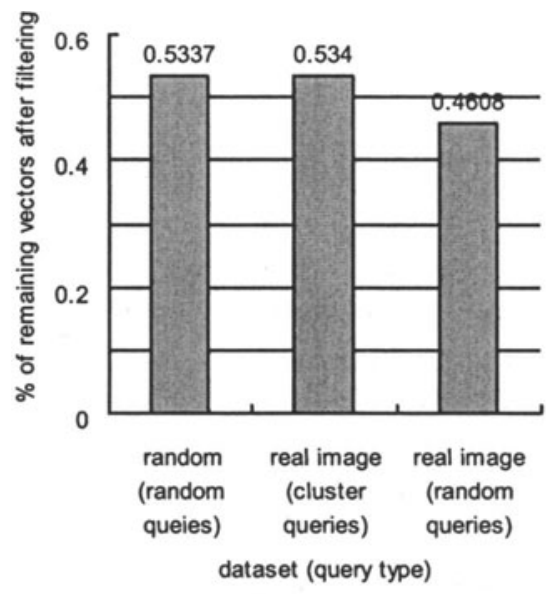

(a) first phase

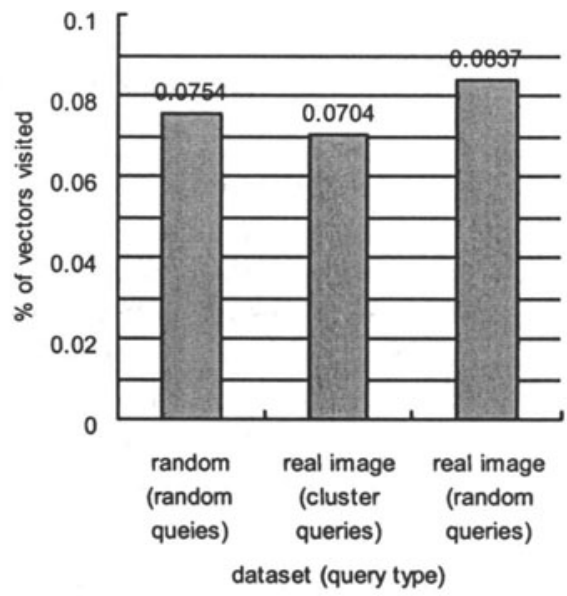

(b) second phase

Figure 6. Selectivity performance of the $\mathrm{LPC}^{+}$-file

\section{PERFORMANCE EVALUATION}

For the experimental evaluation, we used 13,724 256-dimensional image histogram data set described in Section 3. In all experiments, the Euclidean distance metric $L_{2}$ was used, and the number of nearest neighbors to return was always ten, i.e., $k=10$. The page size used in the experiment was $4 \mathrm{~KB}$. 1,000 random and 1000 cluster $10-\mathrm{NN}$ queries were processed and the results were averaged. The initial number of bits per dimension of the approximation cell used was 6 . The density threshold $\tau$ used was 0.001 .

Figure 6 compares the vector selectivity of the $\mathrm{LPC}^{+}$-file for random and real image datasets during the first and the second phases. The $x$-axis represents the datasets and query types used in the experiment. The $y$-axis in Figure 6(a) represents the percentage of remaining vectors (i.e., the vector selectivity) after the first phase and the $y$-axis in Figure 6(b) represents the percentage of real vectors visited during the second phase. Compare the results of Figure 6 with those of Figures 2 and 3. For the LPC ${ }^{+}$-file, the number of remaining vectors in the first phase of the search on the real dataset is less than or nearly equal to that on the random dataset. Observing Figures 2 and 3 for the VA-file and the LPC-file, for the real image dataset, the number of remaining vectors after the first phase is 2.34 to 16.57 times larger than that for the random dataset. In the second phase, for the real image dataset, the $\mathrm{LPC}^{+}$-file visits less real vectors than for the random dataset when the cluster 10 -NN queries were posed, and visits only $0.0083 \%$ more real vectors (actually, the number of vectors read more is 2.79 ) than for the random dataset 
when the random 10-NN queries were posed. On the other hand, for the VAfile and the LPC-file, on the real image dataset, the number of vectors visited during the second phase is 2.02 to 76.77 times larger than that on the random dataset. Summarizing this result, the performance of the LPC ${ }^{+}$-file never degrades even though the dataset is strongly clustered as opposed to the VAfile and the LPC-file.

Figure 7 illustrates the average number of disk accesses during the second phase for 10-NN queries. Note that the disk access pattern is sequential in the first phase, whereas that is random in the second phase. Thus disk accesses occurred in the second phase have greater effects on the search performance than those in the first phase. In Figure 4, we already showed the average number of disk accesses of the $\mathrm{LPC}^{+}$-file, the LPC-file and the VAfile during the first phase. It shows that the number of disk accesses of the LPC $^{+}$-file is almost a half of those of the LPC-file and the VA-file in the first phase. Observing the result of Figure 7, for the $\mathrm{LPC}^{+}$-file, the increase of the number of disk accesses due to the search in the real image dataset is tiny. For cluster queries to a real image dataset, the number of disk accesses is nearly the same as that for a random dataset. For random queries to a real image dataset, in fact, only 2.79 more accesses on the average were performed. On the other hand, for the VA-file and the LPC-file, the increase of the number of disk accesses is significant for the real image dataset, especially for the VA-file. Note that the performance of the LPC ${ }^{+}$-file on the real dataset is far superior to those of the LPC-file and the VA-file.

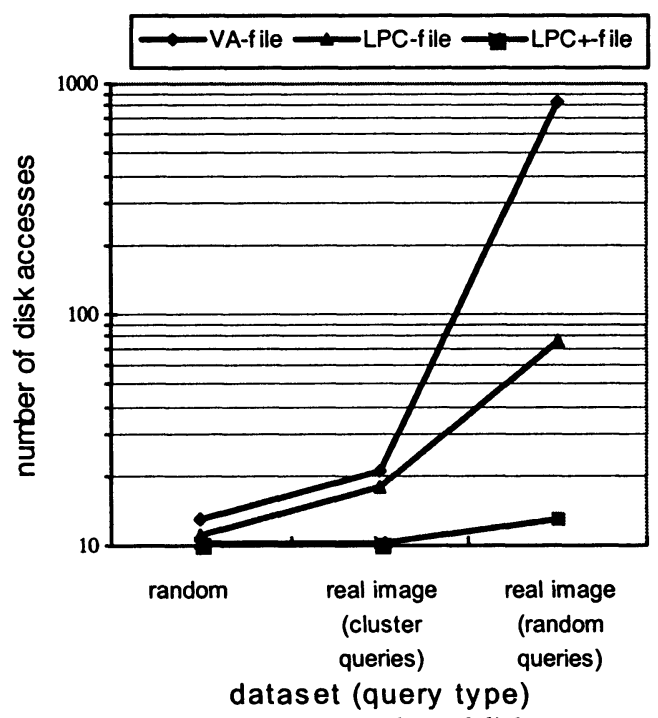

Figure 7. Average number of disk accesses during the second phase 


\section{CONCLUSIONS}

In this paper, we proposed a new approximation-based high-dimensional indexing method called the $\mathrm{LPC}^{+}$-file for clustered image datasets. We stated and discussed the problems associated with the current vector approximation approach for high-dimensional indexing. The current vector approximation approach such as the VA-file and the LPC-file assumes that it is very unlikely that several points lie in the same partition cell, applies a simple space partitioning technique, and employs a uniform bit allocation strategy for the cell representation. However, these assumption and strategies are appropriate only for uniformly distributed datasets, whereas real image datasets are often strongly clustered.

The $\mathrm{LPC}^{+}$-file uses a density-based approach to determine the number of bits to allocate to a cell. The goal of the $\mathrm{LPC}^{+}$-file is to improve the discriminatory (i.e., filtering) power of the vector approximation with the minimum number of bits. The essential idea of the $\mathrm{LPC}^{+}$-file is to assign more bits to vectors in dense regions and less bits to vectors in sparse regions. Moreover, the $\mathrm{LPC}^{+}$-file saves bits to represent the vector approximation by sharing the common bit pattern of the cell on which vectors lie together. The $\mathrm{LPC}^{+}$-file achieves significantly better approximations of the vectors with smaller number of bits, and therefore improves the search performance in clustered high-dimensional image datasets.

\section{REFERENCES}

Arya, S., Mount, D.M., Netanyahu, N.S., Silverman, R., and Wu, A.Y., An Optimal Algorithm for Approximate Nearest Neighbor Searching in Fixed Dimensions, Journal of the $A C M, 45(6), 891-923$, Nov. 1998.

Beckmann, N., Kriegel, H.-P., Schneider, R., and Seeger, B., The R*-tree: An efficient and robust access method for points and rectangles, Proc. of ACM SIGMOD Int'l Conf. on Management of Data, 322-331, 1990.

Berchtold, S., Keim, D.A., Kriegel, H.-P., The X-tree: An Index Structure for HighDimensional Data, Proc. of the Int'l Conf. on Very Large Data Bases, 28-39, 1996.

Cha, G.-H., Zhu, X., Petkovic, D., and Chung, C.-W., An Efficient Indexing Method for Nearest Neighbor Searches in High-Dimensional Image Databases, IEEE Transactions on Multimedia, Vol. 4, No. 1, March 2002.

Cha, G.-H. and Chung, C.-W., A New Indexing Scheme for Content-Based Image Retrieval, Multimedia Tools and Applications, 6(3), 263-288, May 1998.

Chakrabarti, K. and Mehrotra, S., Local Dimensionality Reduction: A New Approach to Indexing High Dimensional Spaces, Proc. of the Int'l Conf. on VLDB, 89-100, 2000. 
Flickner, M., Sawhney, H., Niblack, W., Ashley, J., Huang, Q., Dom, B., Gorkani, M., Hafner, J., Lee, D., Petkovic, D., Steele, D., and Yanker, P., Query by image and video content: the QBIC system, IEEE Computer, 28, 23-32, 1995.

Indyk, P. and Motwani, R., Approximate Nearest Neighbors: Towards Removing the Curse of Dimensionality, Proc. of the ACM Symp. on the Theory of Computing, 604-613, 1998.

Kanth, K.V.R., Agrawal, D. and Singh, A., Dimensionality Reduction for Similarity Searching in Dynamic Databases, Proc. of the ACM SIGMOD Int'l Conf. on Management of Data, 166-176, 1998.

Katayama, N. and Satoh, S. The SR-tree: An Index Structure for High-Dimensional Nearest Neighbor Queries, Proc. of the ACM SIGMOD Int'l Conf. on Management of Data, 369380, 1997.

Kushilevitz, E., Ostrovsky, R. and Y. Rabani, "Efficient Search for Approximate Nearest Neighbor in High Dimensional Spaces," Proc. of the ACM Symposium on the Theory of Computing, 614-623, 1998.

Lin, K.-I., Jagadish, H.V., and Faloutsos, C., The TV-tree: An Index Structure for HighDimensional Data, The VLDB Journal, 3(4), 517-542, 1994.

Megiddo, N. and Shaft, U., Efficient Nearest Neighbor Indexing Based on a Collection of Space-Filling Curves, Technical Report RJ 10093, IBM Almaden Research Center, Nov. 1997.

Niblack, N., Barber, R., Equitz, W., Flickner, M., Glasman, E., Petkovic, D., Yanker, P., Faloutsos, C., and Taubin, G., The QBIC Project: Querying Images By Content Using Color, Texture, and Shape, Proc. of the SPIE Conf. on Storage and Retrieval for Image and Video Databases II, 173-187, 1993.

Shepherd, J., Zhu, X. and Megiddo, N., A Fast Indexing Method for Multidimensional Nearest Neighbor Search, Proc. of the IS\&T/SPIE Conference on Storage and Retrieval for Image and Video Databases VII, 350 - 355, 1999.

Weber, R., Schek, H.-J., and Blott, S., A Quantitative Analysis and Performance Study for Similarity-Search Methods in High-Dimensional Spaces, Proc. of the Int'l Conf. on $V L D B, 194-205,1998$.

\section{BIOGRAPHY}

Guang-Ho Cha received his Ph.D. degree in computer engineering from the Korea Advanced Institute of Science and Technology, Taejon, South Korea in 1997. Since 1997, he has been an Assistant Professor of Multimedia Engineering, Tongmyong Univesity of Information Technology, Busan, South Korea. From 1999 to 2000, he was a Visiting Scientist at the IBM Almaden Research Center, San Jose, CA. His research interests include content-based image/video/music indexing and retrieval, XML and semi-structured databases, and distance learning/education. 